\title{
Radiological analysis of the medial epicondyle in the adolescent throwing athlete
}

\author{
Wuey Min $\underline{N g}^{1}$, Ms(Ortho), Chee Ken $\underline{\text { Chan }}^{1}$, Ms(Ortho), Norimasa Takahashi ${ }^{2}$, MD, Nobuaki Kawai ${ }^{2}$, MD, \\ Kok Kheng $\underline{\text { Teh }}^{1}$, MS(Ortho), $R$ Saravana $^{1}$, BSc, Hiroyuki Sugaya ${ }^{2}$, MD, PhD
}

INTRODUCTION Injuries to the medial structures of the elbow due to overhead throwing games are well documented. However, variations of medial epicondyles are not well described, especially in athletes with fused medial epicondyles. In this study, we evaluated variations in the medial epicondyle of baseball players who were aged 15-17 years and had fused epicondyles.

METHODS In this cross-sectional observational study, 155 skeletally mature baseball players with unilateral medial elbow pain and 310 elbow radiographs were reviewed by two independent reviewers. The medial epicondyles were categorised into three groups: normal, elongated or separated.

RESULTS Among the 155 patients, 65 (41.9\%) had normal epicondyles, 41 (26.5\%) had elongated epicondyles and 49 (31.6\%) had separated epicondyles. The medial epicondyle was larger on the dominant arm for 125 (80.6\%) patients; the mean surface area on the dominant arm was $222.50 \pm 45.77 \mathrm{~mm}^{2}$, while that of the non-dominant arm was $189.14 \pm$ $39.56 \mathrm{~mm}^{2}(\mathrm{p}<0.01)$. Among the three categories of medial epicondyles, separated epicondyles had the largest surface area, followed by elongated and normal epicondyles.

CONCLUSION Medial epicondyles in adolescent throwing athletes can be categorised into three different groups according to their shape (normal, elongated and separated). We observed a correlation between the shape and the surface area of the medial epicondyle in adolescent throwing athletes, with separated medial epicondyles having the largest surface area. Further studies and follow-up are needed to determine the prognostic value and clinical significance of these morphological variations.

Keywords: elbow, injury, Little League, medial epicondyle

\section{INTRODUCTION}

Athletes who engage in overhead throwing games such as baseball, softball, cricket and racquet games are at high risk of repetitive injuries to the elbow. A range of injuries can occur with overhead throwing games, including ulnar collateral ligament (UCL) laxity, avulsion of the medial epicondyle, medial epicondylopathy, posteromedial impingement and osteochondritis dissecans of the capitulum. Repetitive valgus stress, especially during the late cocking and early acceleration phases, may exert a tensile force that exceeds the tensile strength of the $\mathrm{UCL}$, causing compression of the radiocapitellar joint.

The elbow forms the link for load transmission from the hand to the shoulder and, ultimately, to the trunk. The force exerted on the elbow has been estimated to be up to three times that of the person's body weight, with the force peaking at $90^{\circ}$ of elbow flexion. (1) Although the bony configuration is known to be the primary stabiliser of the elbow joint, it only significantly resists the valgus stress when elbow flexion is $<20^{\circ}$ or $>120^{\circ}$. When the elbow is in $20^{\circ}-120^{\circ}$ of flexion, it is the $U C L$ that resists most of the valgus stress. ${ }^{(2)}$ In a throwing athlete, the abnormal repetitive stress over the medial part of the elbow is described as a whipping force. The movement starts with the elbow in acute flexion and ends with it in complete extension, with the forearm in supination or pronation and the wrist in ulnar deviation. During this manoeuvre, a high level of traction force is exerted onto the medial epicondyle, which is the common attachment of the flexor-pronator complex. ${ }^{(3)}$ In the late cocking and early acceleration phase, internal rotation of the shoulder occurs as the forearm is pulled forward at high speed and force; this adds to the valgus stress on the medial structures over the medial epicondyle, and increases the risk of injury in young athletes whose physes are still open and weak.

Little League elbow, which was first described in young baseball players (i.e. young throwing athletes), refers to an injury of the medial structures of the elbow due to repetitive valgus stress. ${ }^{(4)}$ Injuries to the medial structures of the elbow, such as UCL sprains, tears and avulsion, are well documented in the literature. Although Waris described UCL tears in 17 javelin throwers in $1946,{ }^{(5)}$ the diagnosis of and treatment for UCL tears were not well accepted until jobe et al described $U C L$ reconstruction. ${ }^{(3)}$ Since then, there have been many reviews on $U C L$ injury in the literature. ${ }^{(6-8)}$ However, there is a paucity of studies on variations of the medial epicondyle in young throwing athletes. Furthermore, the age range of the athletes included in existing studies was wide, at 9-14 years. The shape and size of the medial epicondyle of very young athletes may still be undergoing changes, as their bones have yet to reach skeletal maturity. Therefore, we examined variations in the medial epicondyle of a group of adolescent athletes aged 15-17 years, whose medial epicondyles had already fused. The data obtained may be a more accurate representation 

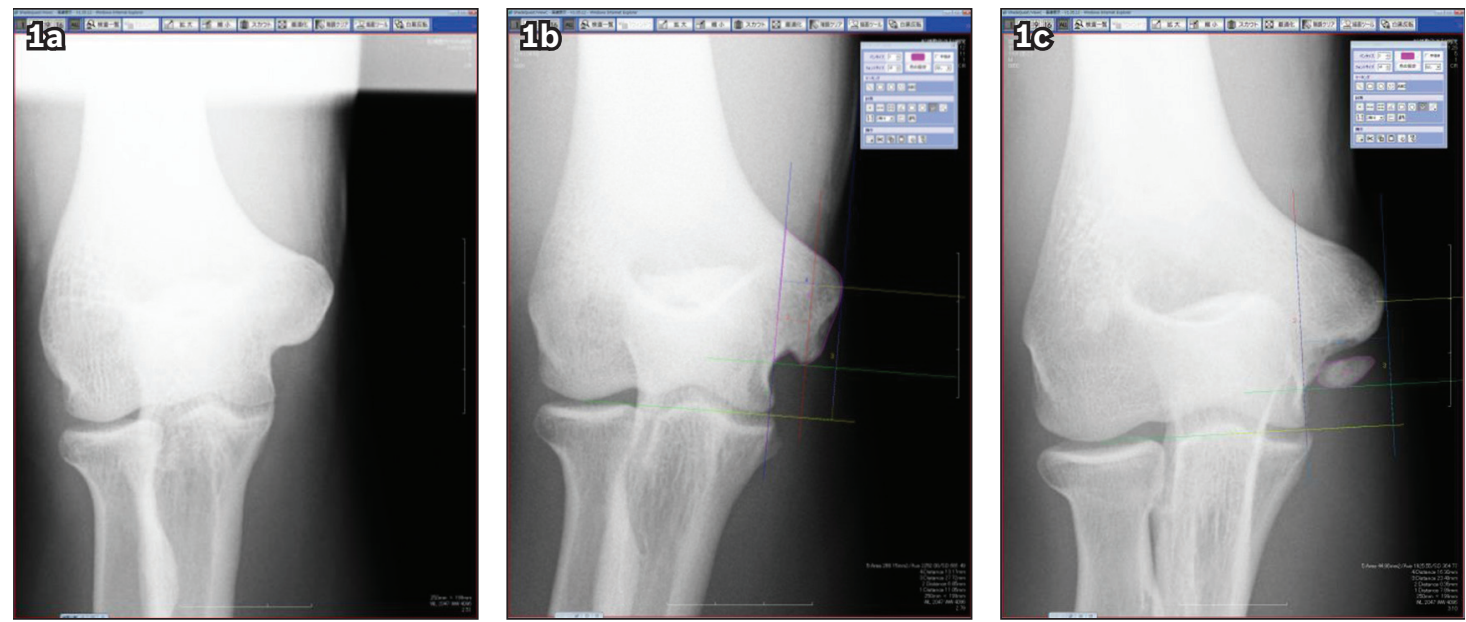

Fig. 1 Radiographs show (a) normal, (b) elongated and (c) separated medial epicondyles.
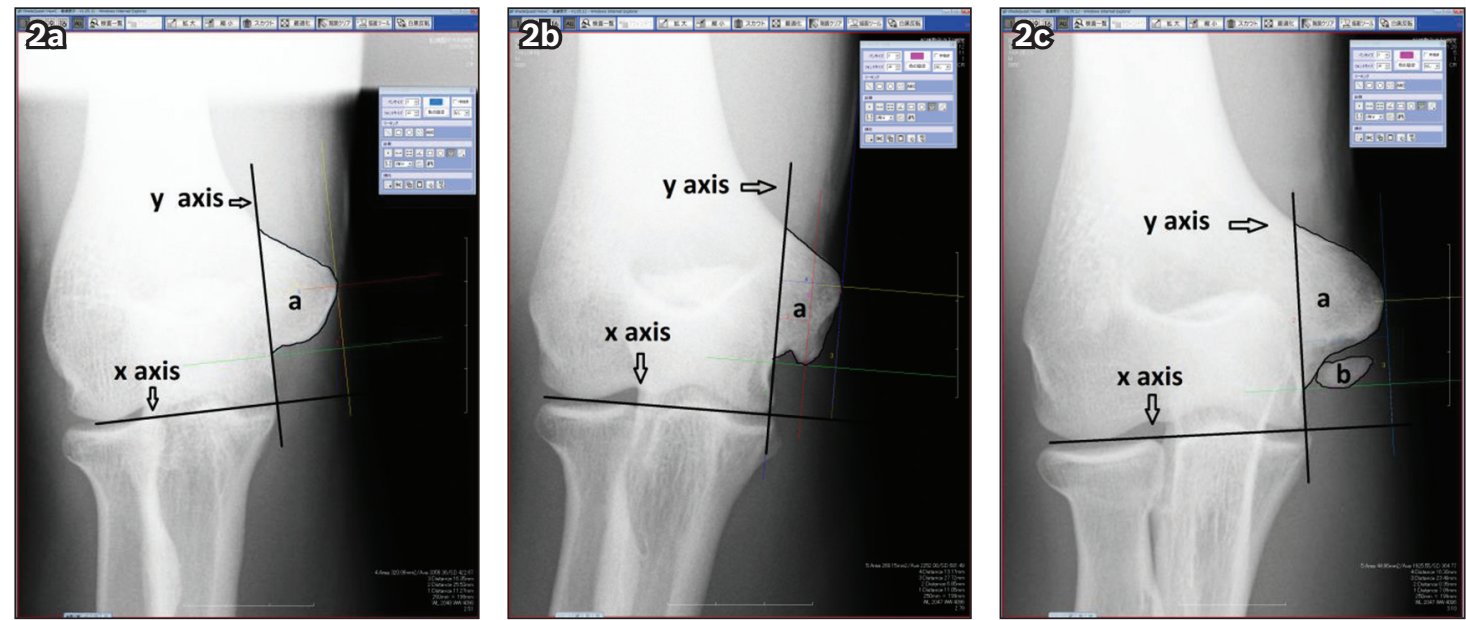

Fig. 2 Radiographs show (a) normal, (b) elongated and (c) separated medial epicondyles and how the measurements were made using the digital radiograph measurement tool. The $x$-axis is parallel to the ulnohumeral and radiocapitellar joint, while the $y$-axis is parallel to the medial border of the trochlea. a: surface area of the medial epicondyle; b: surface area of the separated piece.

of the developmental or pathological variations that are related to Little League elbow.

\section{METHODS}

In this cross-sectional study, we reviewed the radiographs of 203 baseball players who had been treated for medial epicondyle injuries at the Funabashi Orthopaedic Sports Medicine Center, Chiba, Japan. Ethical approval for the study was obtained from the institution. To be included in the study, the patient had to be: (a) a baseball player with medial epicondylar pain; and (b) aged $\geq 15$ years at presentation. Patients with bilateral elbow injury, radiograph(s) taken for only one arm, associated radiocapitellar joint injury, opened physis of the medial epicondyle and/or fracture of the medial condyle were excluded from the study. Younger patients and patients with opened physis were excluded as it would be difficult to determine the actual size and shape of the unossified condyle in this group of patients. Of the 203 patients reviewed, 155 met the inclusion criteria and the radiographs $(n=310)$ of the included patients were analysed. These radiographs of the elbow were in anteroposterior tangential $45^{\circ}$ flexion view, as it best simulates the position of the elbow in the late cocking and early acceleration phase of throwing. It also gives the best view of the medial epicondyle, with the $U C L$ in tension. All radiographs were taken with the cassette located at the back of the elbow and the beam aiming at the elbow joint, perpendicular to the forearm, at a standard distance of $300 \mathrm{~mm}$ from the cassette.

Two senior orthopaedic surgeons who were blinded to the severity of the injury analysed the digital radiographs using YOKOGAWA ShadeQuest ViewR version 1.20 (Yokogawa Medical Solutions Corporation, Tokyo, Japan). The surgeons were first asked to determine the shape of the medial epicondyle based on the radiographic images. The medial epicondyles were categorised into three types: (a) normal - no extra protrusion of bone from the medial epicondyle; (b) elongated - has extra protrusion of bone from the medial epicondyle; or (c) separated - part of the medial epicondyle is separated from the main epicondyle (Fig. 1). The size of the medial epicondyle was measured using its surface area. To quantify the surface area of the medial epicondyle, an x-axis line was drawn along the radiocapitellar and ulnohumeral joint, using the most distal point of the capitulum and trochlea as a guide. A $y$-axis line was then drawn perpendicular to the $\mathrm{x}$-axis line on the medial border of the medial epicondyle (Fig. 2). The outer border of 
the medial epicondyle was then outlined using the odd-shape outliner tool of the radiological software and the area of the medial epicondyle was automatically generated in $\mathrm{mm}^{2}$. The same procedure was repeated for the other elbow of the same patient (i.e. two area measurements were generated for each patient). These measurements were repeated by the same surgeon after two weeks to increase intraobserver reliability. The same procedure was also conducted by the second surgeon to increase interobserver reliability. The four readings obtained were averaged to give the final surface area reading.

Demographic and clinical data (i.e. age, gender, hand dominance and side of injury) were also collected for data analysis. Nonparametric Kappa test was used for descriptive analysis of the shape of the medial epicondyles, while parametric paired sample Student's $t$-test was used to analyse the mean difference between the surface areas of the medial epicondyles of the dominant and non-dominant arms. A p-value $<0.01$ was considered statistically significant.

\section{RESULTS}

A total of 155 pairs of anteroposterior $45^{\circ}$ tangential view radiographs of the elbow $(n=310)$ were analysed by the two surgeons. The radiographs belonged to 155 patients (153 male, 2 female). Their mean age was 15.72 (range 15-17) years. Most of the patients $(n=136)$ were right-hand dominant.

Among the 155 patients, 65 (41.9\%) had normal epicondyles, $41(26.5 \%)$ had elongated epicondyles and 49 (31.6\%) had separated epicondyles (Table I). The surface area of the medial epicondyle was larger on the dominant hand for 125 (80.6\%) patients. The mean surface area of the medial epicondyle of the dominant arm was $222.50 \pm 45.77 \mathrm{~mm}^{2}, 17.6 \%$ larger than that of the non-dominant arm $\left(189.14 \pm 39.56 \mathrm{~mm}^{2}\right)(\mathrm{p}<0.01)$. When the surface areas of the medial epicondyles were analysed according to their shapes, we found that the medial epicondyles were larger on the dominant arm for $93.9 \%, 85.4 \%$ and $67.7 \%$ of the separated, elongated and normal medial epicondyles, respectively (Table I).

The size difference in the medial epicondyles of the dominant and non-dominant arms was most pronounced among the separated medial epicondyles with an average size difference of $29.3 \%$, compared to only $17.9 \%$ among the elongated medial epicondyles and $9.0 \%$ among the normal medial epicondyles (Table II). Among the normal medial epicondyles, the mean surface area on the dominant arm and non-dominant arm was
$205.72 \pm 41.41 \mathrm{~mm}^{2}$ and $188.68 \pm 42.03 \mathrm{~mm}^{2}$, respectively. Among the elongated medial epicondyles, the mean surface area on the dominant arm and non-dominant arm was $233.70 \pm 32.09 \mathrm{~mm}^{2}$ and $198.30 \pm 35.47 \mathrm{~mm}^{2}$, respectively. Among the separated medial epicondyles, the mean surface area on the dominant arm and non-dominant arm was $235.38 \pm 52.67 \mathrm{~mm}^{2}$, and $182.07 \pm 38.64 \mathrm{~mm}^{2}$, respectively.

\section{DISCUSSION}

Since the first description of Little League elbow by Brogdon and Crow in 1960, ${ }^{(4)}$ many reviews and studies have been conducted on the mechanism of injury of the medial structures of the elbow in young athletes. However, most studies focused on UCL injury and $U C L$ reconstruction. ${ }^{(6-8)}$ Adams and Hang et al (in 1965 and 2004 , respectively) showed that there were changes in the medial epicondyles of young throwing athletes. ${ }^{(9,10)}$ In both these studies, the authors evaluated the radiographs of their patients' elbows in anteroposterior view and the age range of their study cohorts were wide, at 9-14 years.

In the present study, we examined the radiographs of 155 baseball players, aged 15-17 years, who presented to our centre with medial elbow pain and instability. The mean age of the patients in our study (15.72 years) is higher than that of the patients from the studies by Adams and Hang et al. ${ }^{(9,10)}$ We specifically chose patients aged 15-17 years as we believed that there would not be any further bony changes to the medial epicondyle during that age range, especially after years of traction injuries to the elbow. With the epiphysis closed, it would be easier to identify all the bony landmarks and thus ensure that measurements of the medial epicondyle are accurate. Anteroposterior $45^{\circ}$ flexion view radiographs of the elbows were used, as the elbow is at maximum valgus stress in the late cocking and early accelerated phase between $30^{\circ}$ and $120^{\circ} .^{(11)}$ In addition, the long axis of the

Table I. Shape of the medial epicondyle of the patients $(n=155)$ according to whether its surface area was larger on the dominant or non-dominant arm.

\begin{tabular}{lcc}
\hline Shape & \multicolumn{2}{c}{ No. (\%) } \\
\cline { 2 - 3 } & $\begin{array}{c}\text { Larger on } \\
\text { dominant arm }\end{array}$ & $\begin{array}{c}\text { Larger on } \\
\text { non-dominant arm }\end{array}$ \\
\hline Normal $(n=65)$ & $44(67.7)$ & $21(32.3)$ \\
Elongated $(n=41)$ & $35(85.4)$ & $6(14.6)$ \\
Separated $(n=49)$ & $46(93.9)$ & $3(6.1)$ \\
Total & $125(80.6)$ & $30(19.4)$ \\
\hline
\end{tabular}

Table II. Size differences between the medial epicondyles of the dominant and non-dominant arm $(n=155)$.

\begin{tabular}{|c|c|c|c|c|c|}
\hline \multirow[t]{3}{*}{ Shape } & \multicolumn{4}{|c|}{ Surface area of the medial epicondyle $\left(\mathrm{mm}^{2}\right)$} & \multirow[t]{3}{*}{ Difference (\%) } \\
\hline & \multicolumn{2}{|c|}{ Dominant arm } & \multicolumn{2}{|c|}{ Non-dominant arm } & \\
\hline & Mean \pm SD & Range & Mean \pm SD & Range & \\
\hline Normal & $205.72 \pm 41.41$ & $130.27-300.81$ & $188.68 \pm 42.03$ & $89.45-284.24$ & 9.0 \\
\hline Elongated & $233.70 \pm 32.09$ & $169.82-310.03$ & $198.30 \pm 35.47$ & $93.12-288.94$ & 17.9 \\
\hline Separated & $235.38 \pm 52.67$ & $147.08-359.55$ & $182.07 \pm 38.64$ & $117.56-273.80$ & 29.3 \\
\hline Overall & $222.50 \pm 45.77$ & $130.77-359.55$ & $189.14 \pm 39.56$ & $89.45-288.94$ & 17.6 \\
\hline
\end{tabular}

SD: standard deviation 
medial epicondyle, UCL and sublime tubercle are all aligned in a straight line at $45^{\circ}$ of elbow flexion.

In the study by Adams, 76 of the 0 pitchers had accelerated growth and separation of the medial epicondyle, and 39 of the 80 pitchers had fragmentation of the medial epicondyle. ${ }^{(9)}$ In Hang et al's study, 94\% (324 out of 343) of the patients had hypertrophy of the medial epicondyle; $57 \%$ had a separation pattern and $19 \%$ had fragmented epicondyles. ${ }^{(10)}$ However, both of these studies were observational in nature and no objective measurements were made.

Among the 155 patients in our study, 65 (41.9\%) patients had normal medial epicondyles, 41 (26.5\%) had elongated medial epicondyles and 49 (31.6\%) had separated medial epicondyles. None of our patients had fragmented medial epicondyles, as all of them had closed physes. In this study, we described a new shape of medial epicondyle, which was labelled as 'elongated medial epicondyle'. In a child with an open physeal plate, the normal-shaped hypertrophy is a bony remodelling process in response to stress. However, in an athlete with an open physeal plate, progressive increase in stress may cause the UCL bony insertion site to detach from the medial epicondyle and become a fragmented piece. In the healing process, some of the fragmented pieces may fuse with the medial epicondyle, which subsequently remodels to form an elongated medial epicondyle. In other cases, some of the fragments may remain separated from the main medial epicondyle and form a separated medial epicondyle. We believe that a medial epicondyle with an elongated or separated shape may contribute to a shortened $U C L$ effective length, making it relatively lax.

We observed that the size difference in the medial epicondyles of the dominant and non-dominant arms was most pronounced among the separated medial epicondyles, followed by the elongated and normal medial epicondyles (29.3\%, 17.9\% and $9.0 \%$, respectively). On average, the medial epicondyle on the dominant arm was $17.6 \%$ larger than the medial epicondyle on the non-dominant arm. Although medial epicondyles were larger on the dominant arm for most of the patients, it was larger on the non-dominant arm for $19.4 \%$ (30 of 155) of the patients. This observation cannot be easily explained by an over-throwing mechanism. Further studies that compare the medial epicondyles of young throwing athletes with those of a normal control group may shed light on these observed variations. One hypothesis is that injury may have caused early physeal closure and growth arrest. We intend to further our study by using magnetic resonance imaging to look at the medial collateral ligament injury, to examine its relationship with the size of the medial epicondyle.

The limitations of the present study were that there was no control group and only two-dimensional measurements of the medial epicondyle could be made using the plain radiographs (i.e. the calculated area may not have been an accurate representation of the size of the medial epicondyle).

To conclude, the medial epicondyles of skeletally mature, adolescent throwing athletes with medial elbow pain can be categorised into three different shapes: normal, elongated and separated. Based on the results of the present study, the shape of the medial epicondyle is correlated to its size. To the best of our knowledge, this is currently the only study that has examined the correlation between the morphological shape and size of the medial epicondyle in adolescent throwing athletes. We observed that the size difference between the medial epicondyle of the dominant arm and the medial epicondyle of the nondominant arm was most pronounced among the separated medial epicondyles, followed by the elongated and normal medial epicondyles. Further studies and follow-up are needed to determine the prognostic value of these morphological variations. The current treatment modalities for injuries to medial structures are mainly conservative and include physical therapies for the correction of shoulder dyskinesis and other functional problems.

\section{REFERENCES}

1. An KN, Hui FC, Morrey BF, Linscheid RL, Chao EY. Muscles across the elbow joint: a biomechanical analysis. J Biomech 1981; 14:659-69.

2. Callaway GH, Field LD, Deng XH, et al. Biomechanical evaluation of the medial collateral ligament of the elbow. J Bone Joint Surg Am 1997; 79:1223-31.

3. Jobe FW, Stark H, Lombardo SJ. Reconstruction of the ulnar collateral ligament in athletes. J Bone Joint Surg Am 1986; 68:1158-63.

4. Brogdon BG, Crow NE. Little leaguer's elbow. Am J Roentgenol Radium Ther Nucl Med 1960; 83:671-5.

5. Waris W. Elbow injuries of javelin-throwers. Acta Chir Scand 1946; 93:563-75.

6. Cain EL Jr, Dugas JR, Wolf RS, Andrews JR. Elbow injuries in throwing athletes: a current concepts review. Am J Sports Med 2003; 31:621-35.

7. Chen FS, Rokito AS, Jobe FW. Medial elbow problems in the overheadthrowing athlete. J Am Acad Orthop Surg 2001; 9:99-113.

8. Safran M, Ahmad CS, Elattrache NS. Ulnar collateral ligament of the elbow. Arthroscopy 2005;21:1381-95.

9. Adams JE. Injury to the throwing arm. A study of traumatic changes in the elbow joints of boy baseball players. Calif Med 1965; 102:127-32.

10. Hang DW, Chao CM, Hang YS. A clinical and roentgenographic study of Little League elbow. Am J Sports Med 2004; 32:79-84.

11. Takahara M, Mura N, Sasaki J, Harada M, Ogino T. Classification, treatment, and outcome of osteochondritis dissecans of the humeral capitellum. J Bone Joint Surg Am 2007; 89:1205-14. 